\title{
Influence of Gravitational Waves on Circular Moving Particles
}

\author{
Manfried Faber, Martin Suda \\ Atominstitut, Technical University Vienna and AIT Austrian Institute of Technology GmbH, Vienna, Austria \\ Email: faber@kph.tuwien.ac.at,martin.suda@ait.ac.at
}

How to cite this paper: Faber, M. and Suda, M. (2018) Influence of Gravitational Waves on Circular Moving Particles. Journal of Modern Physics, 9, 651-668. https://doi.org/10.4236/jmp.2018.94045

Received: February 13, 2018

Accepted: March 26, 2018

Published: March 29, 2018

Copyright (C) 2018 by authors and Scientific Research Publishing Inc. This work is licensed under the Creative Commons Attribution International License (CC BY 4.0).

http://creativecommons.org/licenses/by/4.0/

\begin{abstract}
We investigate the influence of a gravitational wave background on particles in circular motion. We are especially interested in waves leading to stationary orbits. This consideration is limited to circular orbits perpendicular to the incidence direction. As a main result of our calculation, we obtain in addition to the well-known alteration of the radial distance a time dependent correction term for the phase modifying the circular motion of the particle. A background of gravitational waves creates some kind of uncertainty.
\end{abstract}

\section{Keywords}

Gravitational Waves, Circular Orbits, Linearized Einstein Equations

\section{Introduction}

One hundred years after Albert Einstein [1] predicted the existence of gravitational waves and after decades of searches, LIGO has succeeded to detect gravitational waves [2]. Obviously, they originated from a merger of two stellar-mass black holes. With sizes of the interferometer arms of $4 \mathrm{~km}$, LIGO is most sensitive in the frequency band $100-300 \mathrm{~Hz}$. This success of LIGO could support the idea of the existence of gravitational waves in very different frequency ranges. In the Big Bang and in the later history of the universe, a background of gravitational waves [3] could have been produced, still today influencing elementary particles at the atomic scale, as one could speculate. The influence of such waves on particles on circular orbits seems not to have been treated yet. Especially interesting would be an influence on electrons in hydrogen. But apart from this special example, the formalism could be valid quite generally for particles moving in a central force field.

The most important equation of physics of gravitational waves (GW) has been 
derived from general relativity (GR) by A. Einstein himself [1]. It is about radiation power or luminosity of GW and contains the quadrupole tensor. The components of this tensor have been calculated for standard problems by Misner, Thorne and Wheeler [4]. The word-famous pulsar (PSR B1913-16) was the first one to be detected by Taylor and Hulse [5] [6], where a GW-analysis has been executed. The two neutron stars are approaching each other exactly according to the prediction of GR caused by emission of GW. Important properties of this binary star system can be found in [7] [8] [9]. The problem of spin precession has been developed further as well [10]. The binary pulsar system PSR J0737-3039A has been investigated [11] [12] confirming anew GR. Post-Newtonian approximative numerical procedures are used in order to execute computerized simulation of colliding black holes [13] [14] [15] [16] [17]. Ultimately, in 14 September 2015, the first GW (GW150914) from a merger of two black holes had been observed from LIGO [2]. In the attempt of source localization, only large sky patches were identified. Observations of gamma ray bursts seem to be well suited counterparts for the identification of GW sources [18]. A second signal of GW (GW151226) has been observed too [2]. Meanwhile, a numerical model of binary system of 2 stars between 40 and 100 solar masses has been discussed [19]. A binary system of a 3 solar mass black hole with a X-ray star has been identified [20] as well as spinning black holes [21]. Up to now, there have been 7 GW observations, the most recent one GW170817 at 17 August 2017.

Trajectory and precession of spinning particles in external gravitational and electromagnetic fields have been treated analytically in [22] [23] [24] [25]. Impulsive gravitational plane waves, which have a $\delta$-function singularity on a hypersurface, induce a Velocity Memory Effect, as was discussed in [26] [27] [28].

Tiny ripples in spacetime curvature propagate as waves with the speed of light and lead to periodic oscillations in the distance of test particles, as nicely expounded in many books and review articles, e.g., in [29]-[34]. An overview of detection of gravitational waves can be read in [35]. Therein, it is as well described how gravitational waves arise from general relativity. In [36] a foundational review about gravitational waves is given and a critical review of the standard linear approach of the theory is depicted.

Up to now seven gravitational wave events have been detected. These are impressive proofs that gravitational waves really exist. But these special cosmic observations give no information about the spectrum of gravitational waves. They open the possibility that a full spectrum of such waves exists and is up to now unobserved. We are therefore interested to discuss possible consequences of a gravitational wave background. This could have its origin in the big bang similar to the micro-wave background radiation. Our aim is to investigate the possible influence of gravitational waves on particles moving in a central force field. Such external forces beyond gravitation, e.g. electro-magnetic forces, cannot be described by a geodesic equation alone. We have to modify the equation of geodesic deviation by including an additional force term. We are especially inter- 
ested in uncertainties of the orbits and conditions for stationarity. Though gravitational forces between electrons and protons are known to be by 40 orders of magnitude smaller than electric forces we would like to emphasise that crucial for gravitational effects are the amplitudes of gravitational waves. Independent of external forces, the distances between particles are modified by the amplitudes of the gravitational waves and not by the static gravitational forces.

The paper is organized as follows: In Section 2, we repeat the results obtained for distances of test particles in the presence of plane gravitational waves. Afterwards, in Section 3, we discuss an extension of the problem by considering the influence of such gravitational waves on circular moving particles. In Section 4, results of the previous chapter are graphically displayed and discussed. Finally in Section 5 conclusions are drawn.

\section{Motion of Test Particles}

In order to obtain a coordinate independent measure of the wave's influence, the relative motion of two nearby particles can be considered, see e.g., in [29]. It can be described by the geodesic equation in four-dimensional space-time $x^{\mu}=(c t, x, y, z)$. The four-velocity is given by $U^{\mu}=\frac{\mathrm{d} x^{\mu}}{\mathrm{d} \tau}$ and the distance vector $S^{\mu}$ is a solution of the differential equation, see e.g. Equation (52) of [33]

$$
\frac{\mathrm{d}^{2}}{\mathrm{~d} \tau^{2}} S^{\mu}=S^{\sigma} U^{v} U^{\rho} R^{\mu}{ }_{\nu \rho \sigma}
$$

with the Minkowski metric $\eta^{\mu \lambda}=\operatorname{diag}(-1,1,1,1)$ in the flat background. The differential of the proper time $\tau$ is given by $\mathrm{d} \tau=\frac{1}{\gamma} \mathrm{d} t$ with $\gamma=\frac{1}{\sqrt{1-\beta^{2}}}$ and $\beta=\frac{v}{c}$.

In curved space-time the linearized Riemann-tensor $R_{\mu v \rho \sigma}$ is given by

$$
R_{\mu \nu \rho \sigma}=\frac{1}{2}\left(\partial_{\rho} \partial_{\nu} h_{\mu \sigma}+\partial_{\sigma} \partial_{\mu} h_{v \rho}-\partial_{\sigma} \partial_{v} h_{\mu \rho}-\partial_{\rho} \partial_{\mu} h_{v \sigma}\right) .
$$

Here the metric $g_{\mu v}$ has been approximated by $g_{\mu v}=\eta_{\mu v}+h_{\mu v}$ with $\left|h_{\mu v}\right| \ll 1$.

For a gravitational wave $h_{\mu \nu}$, propating in $z$-direction with the velocity of light $\mathcal{c}$, one obtains harmonic oscillations, which neglecting additional phases may be written as (the real part of) plane waves [29]-[34]

$$
h_{\mu v}=C_{\mu \nu} \cos \left(k z-\omega_{g} t\right)
$$

where $\omega_{g}=c k$ is the frequency of the gravitational wave ( $k$ is the wave number). The constant quantities $C_{\mu \nu} \ll 1$ form a symmetric $(0,2)$ tensor with $C_{0 v}=C_{3 v}=0$ and $C_{22}=-C_{11}$ as well as $C_{12}=C_{21}$. They generate a time-varying quadrupole deformation. The two parameters $C_{11}$ and $C_{12}$ are sufficient to describe any quadrupole deformation in the $x y$-plane. In the 
literature waves with $C_{11} \neq 0$ and $C_{12}=0$ are denoted as + polarisations and waves with $C_{11}=0$ and $C_{12} \neq 0$ as $\times$ polarisations [29]-[34]. Any real linear combination of $C_{11} \neq 0 \neq C_{12}$ can be aligned to a + polarisation by a rotation of the coordinate system in the xy-plane. We will therefore restrict our further considerations to linear polarisations in the + mode, see Equation (5) and Equation (8).

Because the Riemann-tensor is first order, the corrections to $U^{v}$ may be ignored, and, for slowly moving particles $\left(\tau=x^{0}=c t\right)$, we have $U^{v}=(1,0,0,0)$, setting $c=1$. As a consequence $R_{\mu 00 \sigma}=\frac{1}{2} \partial_{0}^{2} h_{\mu \sigma}$ and the geodesic Equation (1) becomes

$$
\frac{\partial^{2}}{\partial t^{2}} S^{\mu} \stackrel{(1)}{=} S^{\sigma} \frac{\partial^{2}}{\partial t^{2}} \frac{h^{\mu}}{2} .
$$

$C_{11}$ and $C_{12}$ differ only by a $\pi / 4$-rotation in the xy-plane. Therefore, we can choose without loss of generality $C_{12}=0$ resulting in

$$
\begin{aligned}
& \partial_{0}^{2} S^{1} \stackrel{(4)}{=} S^{1} \partial_{0}^{2} \frac{h_{11}}{2}, \quad \partial_{0}^{2} S^{0^{(4)}}=0, \\
& \partial_{0}^{2} S^{2} \stackrel{(4)}{=}-S^{2} \partial_{0}^{2} \frac{h_{11}}{2}, \quad \partial_{0}^{2} S^{3} \stackrel{(4)}{=} 0,
\end{aligned}
$$

with $h_{11}$ given by Equation (3). For $C_{11} \ll 1$ the solutions of these equations are given in Refs. [29]-[34] by

$$
\begin{aligned}
& S^{1} \approx S_{c}^{1}(t)=S_{c}^{1}(0)\left[1+\frac{h_{11}}{2}\right], \\
& S^{2} \approx S_{c}^{2}(t)=S_{c}^{2}(0)\left[1-\frac{h_{11}}{2}\right], \\
& S^{0}=S^{3}=0 .
\end{aligned}
$$

$S_{c}^{1}(t)$ and $S_{c}^{2}(t)$ are the coordinates of the separation vector in the $x y$-plane. The oscillations in $x$ and $y$ coordinates are $180^{\circ}$ out of phase and lead therefore to linear oscillations around the vector $\left(S_{c}^{1}(0), S_{c}^{2}(0)\right)$, as shown in Figure 1, were we colour-coded the endpoints of the separation vector at each time. To increase the visibility we have chosen an unrealistic large value for $C_{11}$. The functions in Equation (6) fulfil Equation (5) ${ }^{1}$

$$
\begin{aligned}
& S_{c}^{1}(0)\left(-\omega_{g}^{2}\right) \frac{h_{11}}{2} \stackrel{(5)}{\approx} S_{c}^{1}(t)\left(-\omega_{g}^{2}\right) \frac{h_{11}}{2}, \\
& S_{c}^{2}(0) \omega_{g}^{2} \frac{h_{11}}{2} \stackrel{(5)}{\approx}-S_{c}^{2}(t)\left(-\omega_{g}^{2}\right) \frac{h_{11}}{2},
\end{aligned}
$$

\section{Circular Motion with Constant Angular Velocity}

Let us consider a light particle rotating in the xy-plane on a circle with radius $r$ with constant angular velocity $\omega$ around a heavy particle, like in Bohr's model for the hydrogen atom. We assume that the radius $r$ and the azimuthal angle

${ }^{1}$ With the approximation symbol " $\approx$ " we indicate accuracy up to order $C_{11}$. 


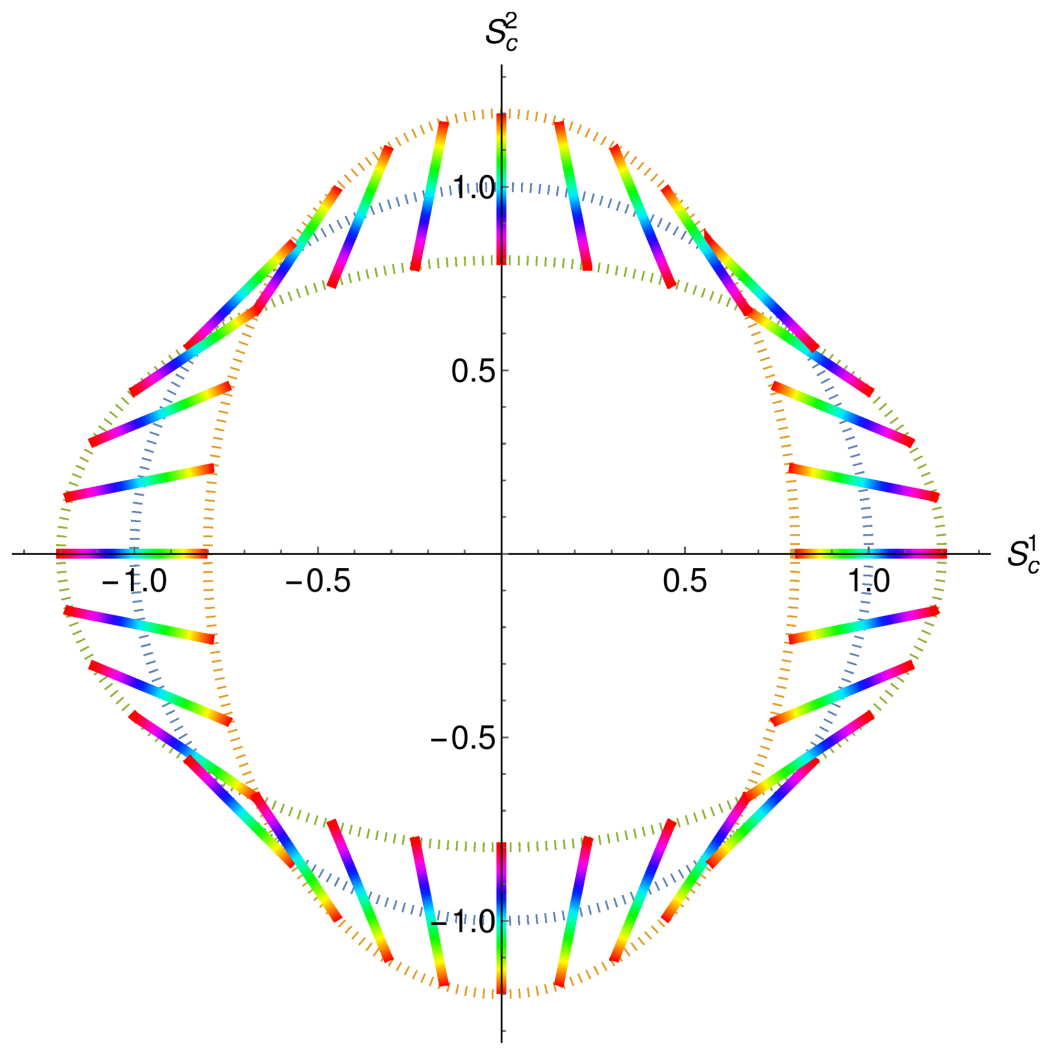

Figure 1. Linear oscillations of $\left(S_{c}^{1}(t), S_{c}^{2}(t)\right)$ with time $t$ according to Equation (6) for various values of $S_{c}^{1}(0)$ and $S_{c}^{2}(0)$ with $\sqrt{\left[S_{c}^{1}(0)\right]^{2}+\left[S_{c}^{2}(0)\right]^{2}}=1$ and $C_{11}=0.2$. The endpoints of the separation vector at each time are colour-coded.

$\varphi=\omega t$ of this motion are slightly disturbed by a gravitational wave with + polarisation incident in 3-direction with $h_{\mu v}$ given by Equation (3) and the symmetric tensor

$$
C_{0 v}=C_{12}=C_{3 v}=0, \quad C_{22}=-C_{11} .
$$

In a generalised equation of motion we have to extend Equation (1) which takes into account the gravitational force on the rhs by a central force. This central force keeps the light particle on a circle. For the assumed circular motion this force can be approximated by the expression for the centripetal force

$$
\partial_{0}^{2} S^{\mu}=S^{\sigma} \frac{U^{v}}{\gamma} \frac{U^{\rho}}{\gamma} R^{\mu}{ }_{\nu \rho \sigma}+\left(0, \boldsymbol{a}_{r}, 0\right)^{\mu},
$$

where $\boldsymbol{a}_{r}$ is the radial acceleration in the xy-plane caused by the external centripetal force. The left hand side of Equation (9), the acceleration acting on the particle, originates therefore in the curvature of space-time introduced by the gravitational wave, see Equation (1), and in the external central force approximated in the second term on the right hand side of Equation (9) by the corresponding centripetal force.

The geometrical term $S^{\sigma} U^{v} U^{\rho} R^{\mu}{ }_{\nu \rho \sigma}$ representing the curved space-time due 
to gravitational waves simplifies drastically due to our special assumptions (3) and (8)-wave propagating in $z$-direction and particle rotating in the xy-plane. $h_{\mu v}$ of Equation (3) depends only on $z$ and $t$. In the curvature tensor $R_{\mu v \rho \sigma}$ of Equation (2) the derivatives of $h_{\mu v}$ are always contracted either with $S^{\mu}$ or $U^{\mu}$. Due to $S^{3}=U^{3}=0$ finally only time derivatives of $h_{11}$ and $h_{22}=-h_{11}$ matter. Therefore, the contributions of the geometrical term read

$$
\begin{aligned}
& S^{\sigma} U^{v} U^{\rho} R_{v \rho \sigma}^{0} \stackrel{(2)}{=}-\partial_{0}^{2} \frac{h_{11}}{2}\left[S^{0}\left(U^{1} U^{1}-U^{2} U^{2}\right)-S^{1} U^{0} U^{1}+S^{2} U^{0} U^{2}\right], \\
& S^{\sigma} U^{v} U^{\rho} R_{v \rho \sigma}^{1} \stackrel{(2)}{=} \partial_{0}^{2} \frac{h_{11}}{2}\left[-S^{0} U^{0} U^{1}+S^{1} U^{0} U^{0}\right], \\
& S^{\sigma} U^{v} U^{\rho} R_{v \rho \sigma}^{2} \stackrel{(2)}{=} \partial_{0}^{2} \frac{h_{11}}{2}\left[S^{0} U^{0} U^{2}-S^{2} U^{0} U^{0}\right] .
\end{aligned}
$$

As expected, due to the factor

$$
\partial_{0}^{2} \frac{h_{11}}{2}=-\frac{C_{11}}{2} \omega_{g}^{2} \cos \left(k z-\omega_{g} t\right) .
$$

all these contributions are of first order in $C_{11}$.

In the spirit of the approximate solution (6) we expect that the distance vector of pure circular motion indicated by the subscript 0

$$
S_{0}^{\mu}=(0, r \cos \varphi, r \sin \varphi, 0), \quad \varphi(t)=\omega t,
$$

is modified by terms linear in $C_{11}$. The modifications of the radial distance according to Equation (5) lead to Coriolis forces inducing variations of the angular velocity. Therefore, we try the ansatz

$$
\begin{gathered}
S^{0}=S^{0}(t), \\
S^{1}=R_{1}(t) \cos \varphi_{1}(t)=r\left[1+\frac{h_{11}(t)}{2}\right] \cos \left[\varphi(t)-\eta_{1}(t)\right], \\
S^{2}=R_{2}(t) \sin \varphi_{2}(t)=r\left[1-\frac{h_{11}(t)}{2}\right] \sin \left[\varphi(t)+\eta_{2}(t)\right], \\
S^{3}=0,
\end{gathered}
$$

where $S^{0}, \eta_{1}$ and $\eta_{2}$ are of first order in $C_{11}$. For the velocity vector $U^{\mu}$ we get

$$
\begin{gathered}
\frac{U^{0}}{\gamma}=1+\partial_{0} S^{0}(t), \\
\frac{U^{1}}{\gamma}=\partial_{0} S^{1} \stackrel{(14)}{=} \partial_{0} R_{1} \cos \varphi_{1}-R_{1} \sin \varphi_{1} \dot{\varphi}_{1}, \\
\frac{U^{2}}{\gamma}=\partial_{0} S^{2} \stackrel{(15)}{=} \partial_{0} R_{2} \sin \varphi_{2}+R_{2} \cos \varphi_{2} \dot{\varphi}_{2}, \\
U^{3}=\partial_{0} S^{3} \stackrel{(16)}{=} 0
\end{gathered}
$$

using dots for time-derivatives $\partial_{0}$. With Equations (13)-(20) we are ready to calculate the geometrical terms (10) 


$$
\begin{gathered}
S^{\sigma} \frac{U^{v}}{\gamma} \frac{U^{\rho}}{\gamma} R_{\nu \rho \sigma}^{0} \stackrel{(10)}{\approx} \\
\approx-\partial_{0}^{2} \frac{h_{11}}{2}\left[-S_{0}^{1} \partial_{0} S_{0}^{1}+S_{0}^{2} \partial_{0} S_{0}^{2}\right] \stackrel{(12)}{\approx} \\
\approx-\partial_{0}^{2} \frac{h_{11}}{2} r^{2} \omega \sin (2 \varphi) \stackrel{(11)}{=} \\
\stackrel{(12)}{=} \frac{C_{11}}{2} \omega_{g}^{2} \cos \left(k z-\omega_{g} t\right) r^{2} \omega \sin (2 \omega t), \\
S^{\sigma} \frac{U^{v}}{\gamma} \frac{U^{\rho}}{\gamma} R_{\nu \rho \sigma}^{1} \stackrel{(10)}{\approx} \partial_{0}^{2} \frac{h_{11}}{2} S^{1} \stackrel{(14)}{=} \partial_{0}^{2} R_{1} \cos \varphi_{1}, \\
S^{\sigma} \frac{U^{v}}{\gamma} \frac{U^{\rho}}{\gamma} R_{\nu \rho \sigma}^{2} \stackrel{(10)}{\approx}-\partial_{0}^{2} \frac{h_{11}}{2} S^{2} \stackrel{(15)}{=} \partial_{0}^{2} R_{2} \sin \varphi_{2} .
\end{gathered}
$$

Thus, the equation of motion (9) for the time-component reads

$$
\partial_{0}^{2} S^{0} \stackrel{(21)}{\approx} \frac{C_{11}}{4} r^{2} \omega \omega_{g}^{2}\left\{\sin \left[\left(2 \omega-\omega_{g}\right) t+k z\right]+\sin \left[\left(2 \omega+\omega_{g}\right) t-k z\right]\right\}
$$

with the solution

$$
S^{0 \stackrel{(24)}{\approx}}-\frac{C_{11}}{4} r^{2} \omega \omega_{g}^{2}\left\{\frac{\sin \left[\left(2 \omega-\omega_{g}\right) t+k z\right]}{\left(2 \omega-\omega_{g}\right)^{2}}+\frac{\sin \left[\left(2 \omega+\omega_{g}\right) t-k z\right]}{\left(2 \omega+\omega_{g}\right)^{2}}\right\} .
$$

More terms we get for the $x$ - and $y$-components

$$
\begin{gathered}
\partial_{0}^{2} S^{1} \stackrel{(14)}{=} \partial_{0}^{2} R_{1} \cos \varphi_{1}-2 \partial_{0} R_{1} \sin \varphi_{1} \dot{\varphi}_{1}-R_{1} \cos \varphi_{1} \dot{\varphi}_{1}^{2}-R_{1} \sin \varphi_{1} \ddot{\varphi}_{1}, \\
\partial_{0}^{2} S^{2} \stackrel{(15)}{=} \partial_{0}^{2} R_{2} \sin \varphi_{2}+2 \partial_{0} R_{2} \cos \varphi_{2} \dot{\varphi}_{2}-R_{2} \sin \varphi_{2} \dot{\varphi}_{2}^{2}+R_{2} \cos \varphi_{2} \ddot{\varphi}_{2} .
\end{gathered}
$$

To simplify the notation for the four terms on the rhs we use two-dimensional vectors in the xy-plane. Further on we denote them by $\boldsymbol{a}_{1}, \boldsymbol{a}_{2}, \boldsymbol{a}_{3}$ and $\boldsymbol{a}_{4}$ and use dots for time derivatives $\partial_{0}$. We realise that the first terms $\boldsymbol{a}_{1}$ in these two equations are the geometrical terms (22) and (23). The third terms

$$
\boldsymbol{a}_{3}=-\left(\begin{array}{l}
R_{1} \cos \varphi_{1} \dot{\varphi}_{1}^{2} \\
R_{2} \sin \varphi_{2} \dot{\varphi}_{2}^{2}
\end{array}\right) \stackrel{(14)}{=}-\left(\begin{array}{l}
S_{1} \dot{\varphi}_{1}^{2} \\
S_{2} \dot{\varphi}_{2}^{2}
\end{array}\right)
$$

include the centrifugal acceleration $\boldsymbol{a}_{r}$ acting in radial direction $\boldsymbol{S}=\left(\begin{array}{c}S^{1} \\ S^{2}\end{array}\right)$, see Figure 2,

$$
\boldsymbol{a}_{r}=\frac{\boldsymbol{a}_{3} \boldsymbol{S}}{\boldsymbol{S}^{2}} \boldsymbol{S}=-\frac{\left(S^{1}\right)^{2} \dot{\varphi}_{1}^{2}+\left(S^{2}\right)^{2} \dot{\varphi}_{2}^{2}}{\left(S^{1}\right)^{2}+\left(S^{2}\right)^{2}} \boldsymbol{S}
$$

Inserting

$$
\begin{aligned}
& \dot{\varphi}_{1}^{2} \stackrel{(14)}{=}\left(\dot{\varphi}-\dot{\eta}_{1}\right)^{2} \stackrel{(14)}{\approx} \omega^{2}-2 \omega \dot{\eta}_{1}, \\
& \dot{\varphi}_{2}^{2} \stackrel{(14)}{=}\left(\dot{\varphi}+\dot{\eta}_{2}\right)^{2} \stackrel{(14)}{\approx} \omega^{2}+2 \omega \dot{\eta}_{2},
\end{aligned}
$$

we get 


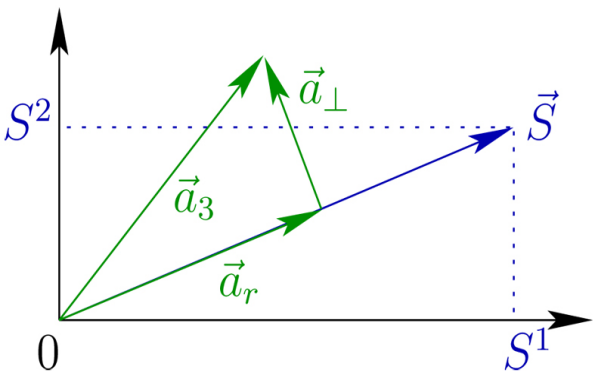

Figure 2. Decomposition of the acceleration $\boldsymbol{a}_{3}$ in components parallel and perpendicular to the distance vector $S$ in the xy-plane.

$$
\begin{gathered}
\boldsymbol{a}_{r} \stackrel{(29)}{=}-\omega^{2} \boldsymbol{S}\left[1-\frac{2}{\omega} \frac{\dot{\eta}_{1}\left(S^{1}\right)^{2}-\dot{\eta}_{2}\left(S^{2}\right)^{2}}{\boldsymbol{S}^{2}}\right] \\
\stackrel{(14)}{=}-\omega^{2} \boldsymbol{S}\left[1-\frac{2}{\omega}\left(\dot{\eta}_{1} \cos ^{2} \varphi-\dot{\eta}_{2} \sin ^{2} \varphi\right)\right] .
\end{gathered}
$$

The remainder of $\boldsymbol{a}_{3}$

$$
\begin{gathered}
\boldsymbol{a}_{\perp}=\boldsymbol{a}_{3}-\boldsymbol{a}_{r} \underset{(31)}{\stackrel{(28)}{=}} 2 \omega\left(\begin{array}{c}
S^{1}\left[\dot{\eta}_{1}-\dot{\eta}_{1} \cos ^{2} \varphi+\dot{\eta}_{2} \sin ^{2} \varphi\right] \\
S^{2}\left[-\dot{\eta}_{2}-\dot{\eta}_{1} \cos ^{2} \varphi+\dot{\eta}_{2} \sin ^{2} \varphi\right]
\end{array}\right) \\
\stackrel{(14)}{=} 2 \omega\left(\begin{array}{c}
S^{2}\left(\dot{\eta}_{1}+\dot{\eta}_{2}\right) \sin \varphi \cos \varphi \\
-S^{1}\left(\dot{\eta}_{1}+\dot{\eta}_{2}\right) \sin \varphi \cos \varphi
\end{array}\right)
\end{gathered}
$$

contributes to the azimuthal accelerations together with the second and forth term in Equations (26) and (27). Since there are no forces leading to azimuthal accelerations we have to set their sum to zero

$$
\underbrace{\boldsymbol{a}_{3}-\boldsymbol{a}_{r}}_{\boldsymbol{a}_{\perp}}+\boldsymbol{a}_{2}+\boldsymbol{a}_{4} \stackrel{!}{=} 0 .
$$

This is a condition for the time dependence of the angular velocity. It leads to

$$
\begin{aligned}
& \omega \sin (2 \varphi)\left(\dot{\eta}_{1}+\dot{\eta}_{2}\right)-\omega \partial_{0} h_{11}+\ddot{\eta}_{1}=0, \\
& -\omega \sin (2 \varphi)\left(\dot{\eta}_{1}+\dot{\eta}_{2}\right)-\omega \partial_{0} h_{11}+\ddot{\eta}_{2}=0 .
\end{aligned}
$$

The sum of these two equations

$$
\ddot{\eta}_{1}+\ddot{\eta}_{2} \stackrel{(34)}{\stackrel{(35)}{=}} 2 \omega \partial_{0} h_{11}
$$

can be solved by

$$
\dot{\eta}_{1}+\dot{\eta}_{2} \stackrel{(36)}{=} 2 \omega h_{11}
$$

and by

$$
\eta_{1}+\eta_{2} \stackrel{(3)}{=}-2 C_{11} \frac{\omega}{\omega_{g}} \sin \left(k z-\omega_{g} t\right) .
$$

The difference of the two Equations (34) and (35) 


$$
\begin{aligned}
& \ddot{\eta}_{2}-\ddot{\eta}_{1} \underset{(35)}{\stackrel{(34)}{=}} 2 \omega \sin (2 \varphi)\left(\dot{\eta}_{1}+\dot{\eta}_{2}\right) \stackrel{(37)}{=} 4 \omega^{2} \sin (2 \varphi) h_{11} \\
& \stackrel{(3)}{=} 4 C_{11} \omega^{2} \sin (2 \varphi) \cos \left(k z-\omega_{g} t\right)
\end{aligned}
$$

can be solved by

$$
\eta_{2}-\eta_{1} \stackrel{(39)}{=} 2 C_{11} \omega^{2}\left\{\frac{\sin \left[\left(2 \omega-\omega_{g}\right) t+k z\right]}{\left(2 \omega-\omega_{g}\right)^{2}}+\frac{\sin \left[\left(2 \omega+\omega_{g}\right) t-k z\right]}{\left(2 \omega+\omega_{g}\right)^{2}}\right\} .
$$

We get the results

$$
\begin{aligned}
& \eta_{1,2} \stackrel{(38)}{=}-C_{11}\left\{\frac{\omega}{\omega_{g}} \sin \left(k z-\omega_{g} t\right)\right. \\
&\left. \pm \omega^{2}\left[\frac{\sin \left[\left(2 \omega-\omega_{g}\right) t+k z\right]}{\left(2 \omega-\omega_{g}\right)^{2}}+\frac{\sin \left[\left(2 \omega+\omega_{g}\right) t-k z\right]}{\left(2 \omega+\omega_{g}\right)^{2}}\right]\right\} \\
& \stackrel{(25)}{=} \frac{\omega}{\omega_{g}}\left\{-C_{11} \sin \left(k z-\omega_{g} t\right) \pm \frac{4 S^{0}}{\omega_{g} r^{2}}\right\},
\end{aligned}
$$

where according to Equation (25) $S^{0}$ is of order $C_{11}$.

Result: We have obtained the time-dependent correction terms $\eta_{1,2}=\eta_{1,2}(t)$ which have to be subtracted from (added to) $\varphi(t)=\omega t$ in Equations (14) and (15) when circular moving particles are influenced by gravitational waves, Equation (3). We have found a solution $S^{\mu}$ according to the ansatz (13)-(16) fullfilling the equation of motion (9).

The distance vector $\boldsymbol{S}=\left(S^{1}, S^{2}\right)$ from the first to the second particle, shown in Figure 2, can be represented by the polar coordinates

$$
R=\sqrt{\left(S^{1}\right)^{2}+\left(S^{2}\right)^{2}}, \quad \phi=\arctan \frac{S^{2}}{S^{1}}
$$

with $S^{1}$ and $S^{2}$ given in Equations (14) and (15).

The gravitational wave modifies the radius $R$ of the circular motion and in consequence the angular velocity deviates from $\omega$.

\section{Results and Discussion}

Gravitational waves from a background of gravitational waves with frequencies $\omega_{g}$ much smaller than the frequencies $\omega$ of orbital motion lead to periodic shifts of attractive two-particle systems and do not influence their radial distance $R=r$. Significant modifications of circular orbits appear only, if $\omega_{g}$ and $\omega$ are of the same order of magnitude, see Figures 3-12, where figures of the orbits are displayed evaluating Expressions (14) and (15) for $S^{1}$ and $S^{2}$. Since we want to compare the positions of the two particles at equal times we put $S_{0}=0$ in Equation (41). To allow for better visibility we choose the amplitudes of the quadrupole oscillations in most diagrams unrealistically large, $C_{11} / 2=0.05$. Only in Figure 6 and Figure 10 we choose slightly smaller values.

For integer ratios $\omega_{g} / \omega$ the orbits get periodic and close after one revolution 


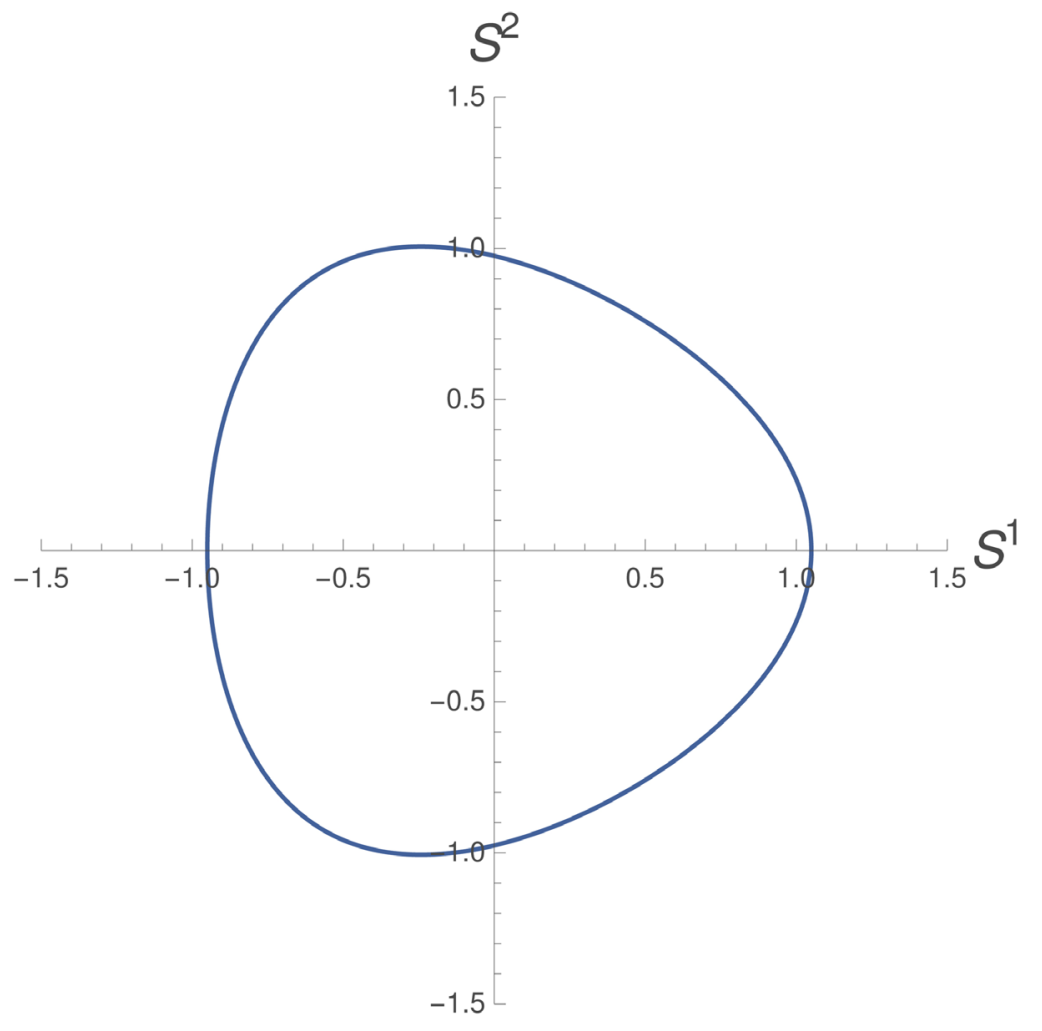

Figure 3. $\frac{C_{11}}{2}=0.05, k z=0, \frac{\omega_{g}}{\omega}=1, \quad t=T$.

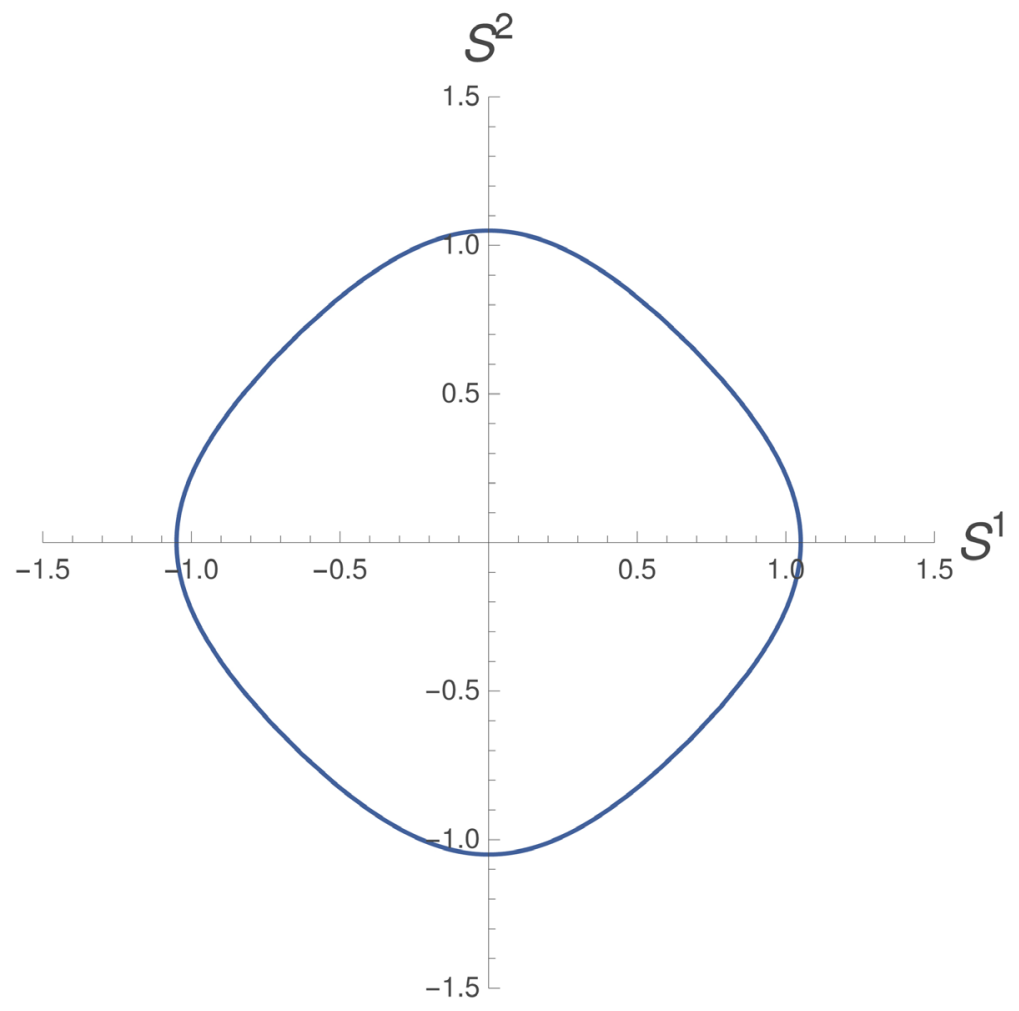

Figure 4. $\frac{C_{11}}{2}=0.05, k z=0, \frac{\omega_{g}}{\omega}=2, \quad t=T$. 


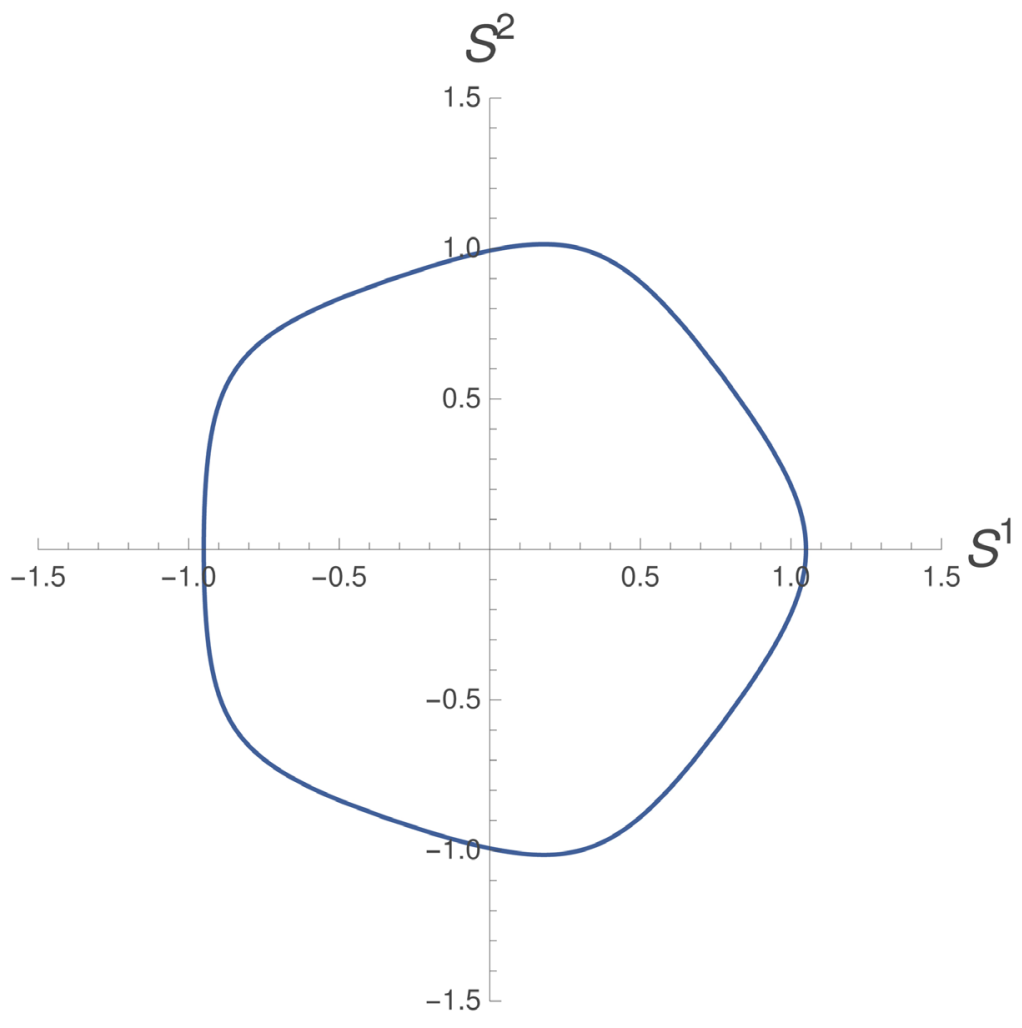

Figure 5. $\frac{C_{11}}{2}=0.05, k z=0, \frac{\omega_{g}}{\omega}=3, t=T$.

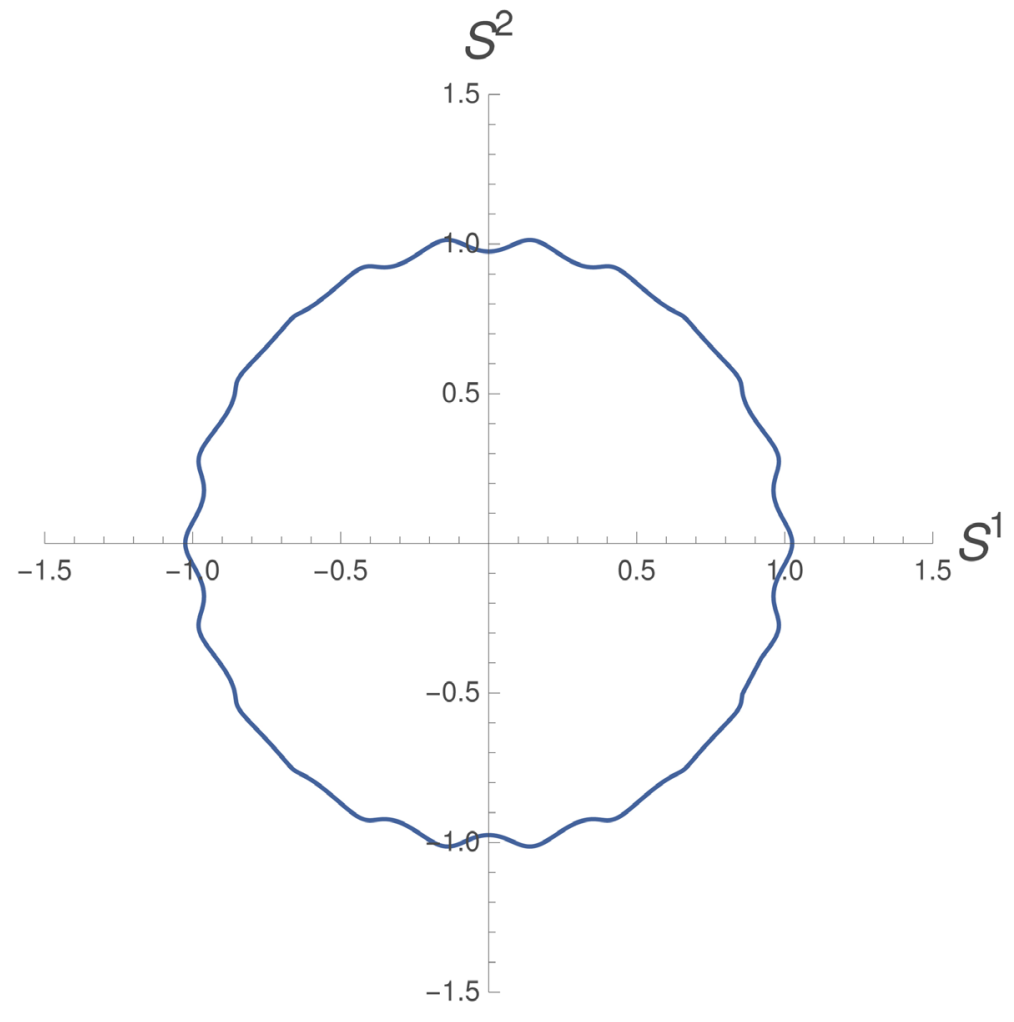

Figure 6. $\frac{C_{11}}{2}=0.025, k z=0, \frac{\omega_{g}}{\omega}=20, t=T$. 


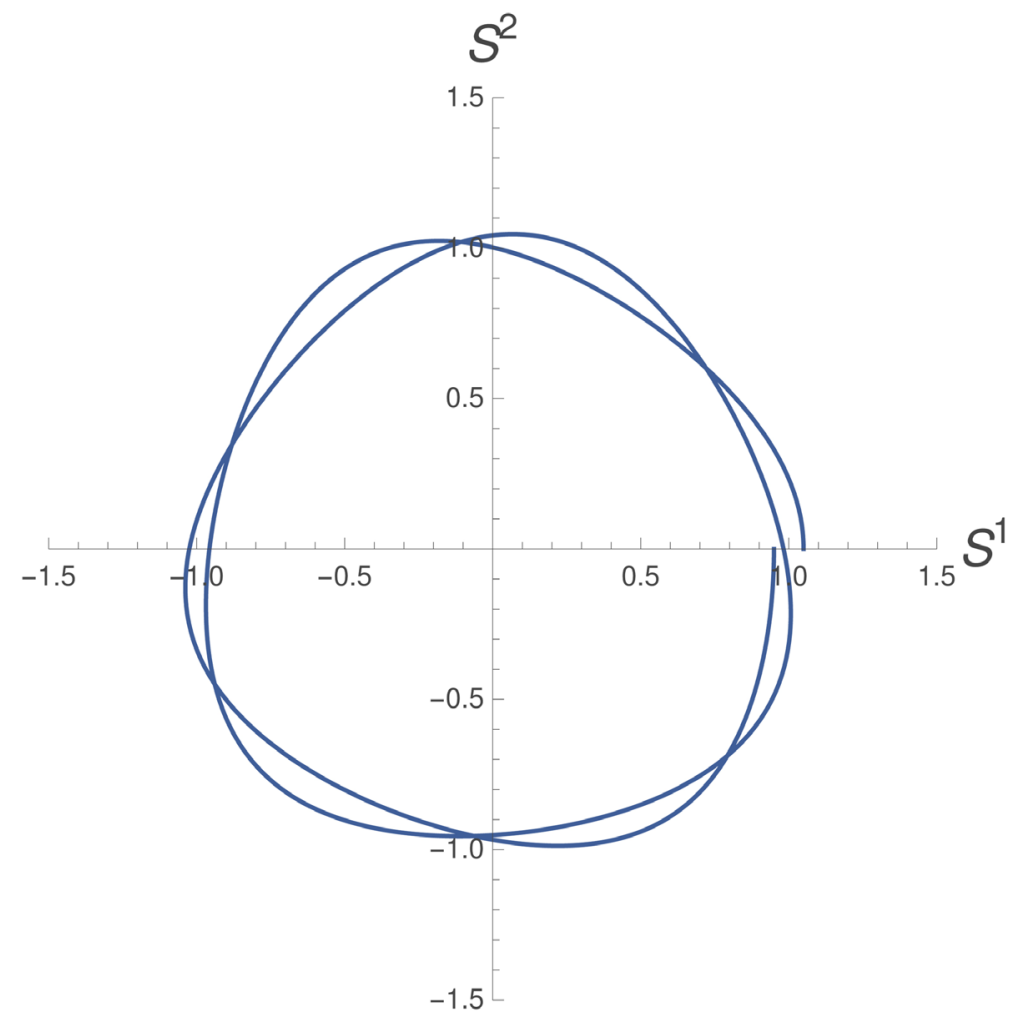

Figure 7. $\frac{C_{11}}{2}=0.05, k z=0, \frac{\omega_{g}}{\omega}=1.25, t=2 T$.

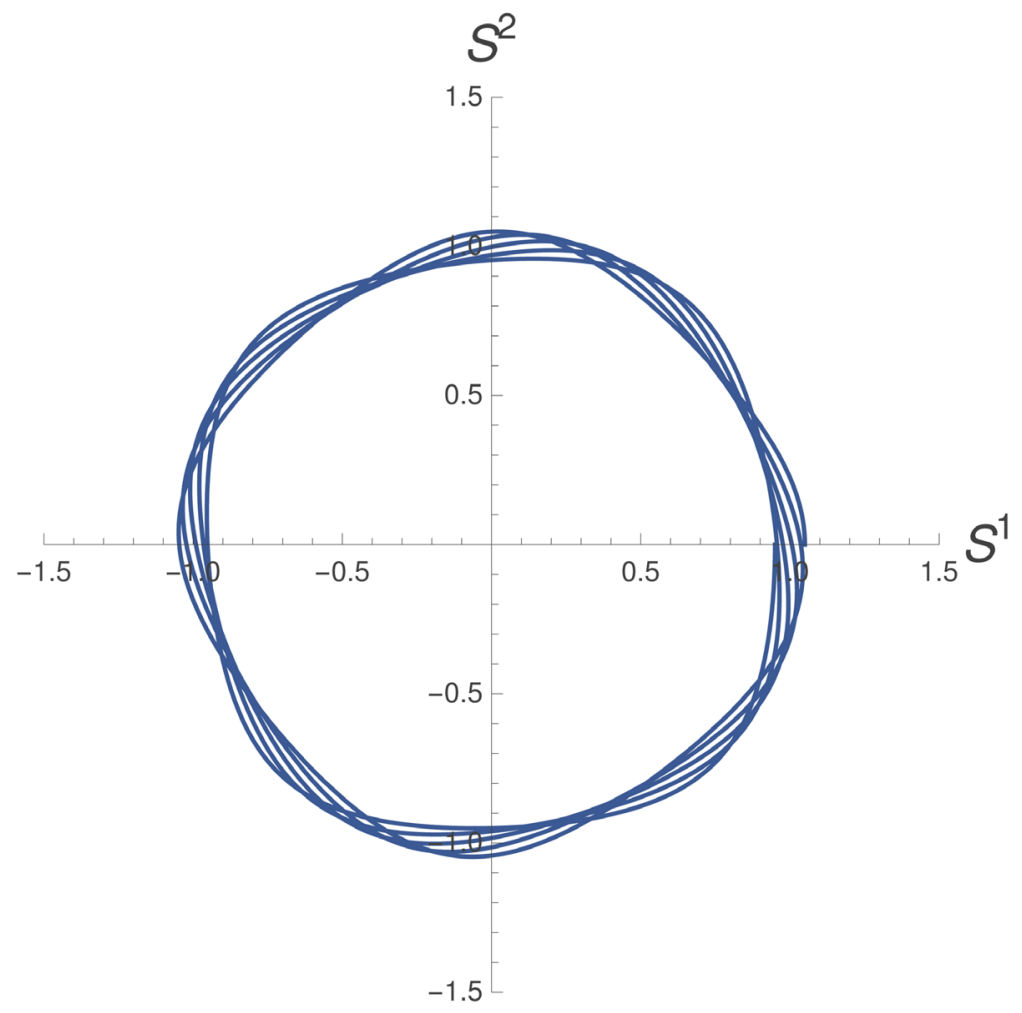

Figure 8. $\frac{C_{11}}{2}=0.05, k z=0, \frac{\omega_{g}}{\omega}=2.1, t=5 T$. 


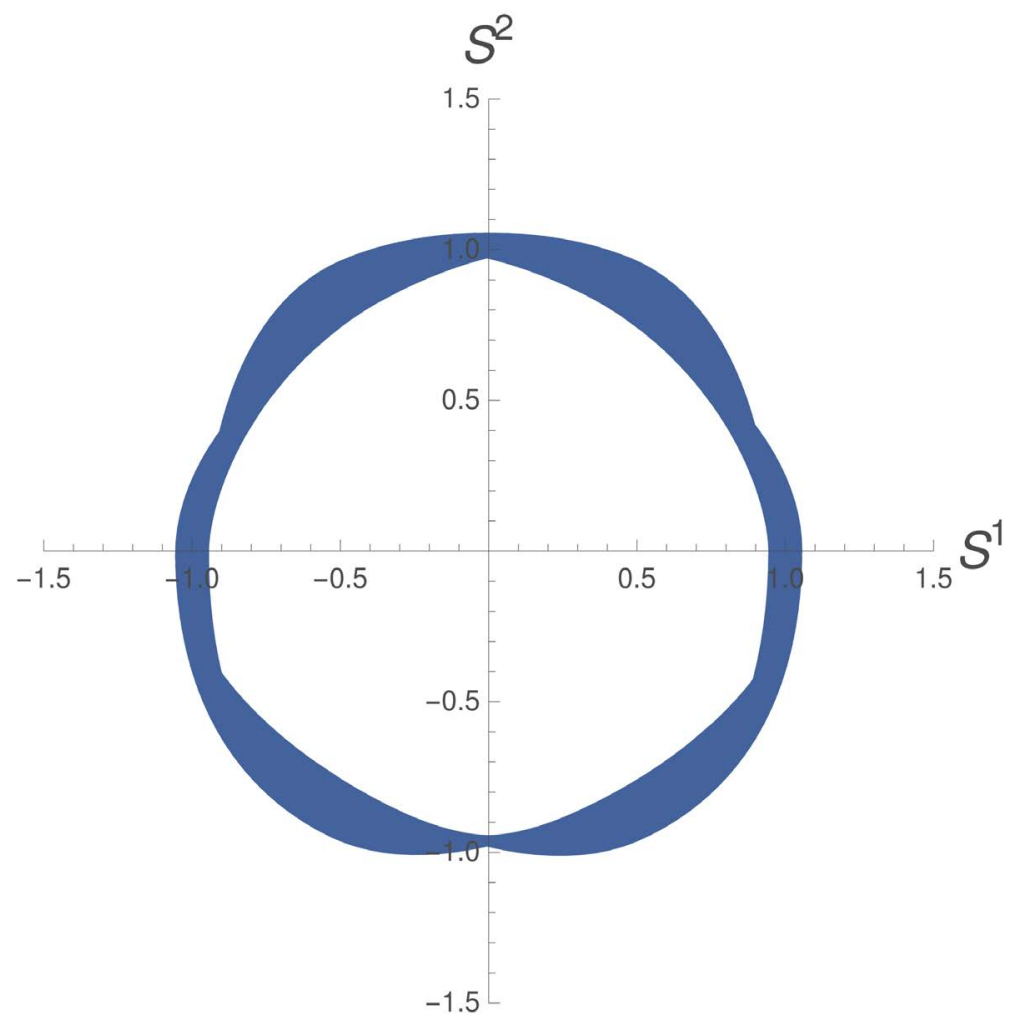

Figure 9. $\frac{C_{11}}{2}=0.05, k z=0, \frac{\omega_{g}}{\omega}=1.02, t=25 T$.

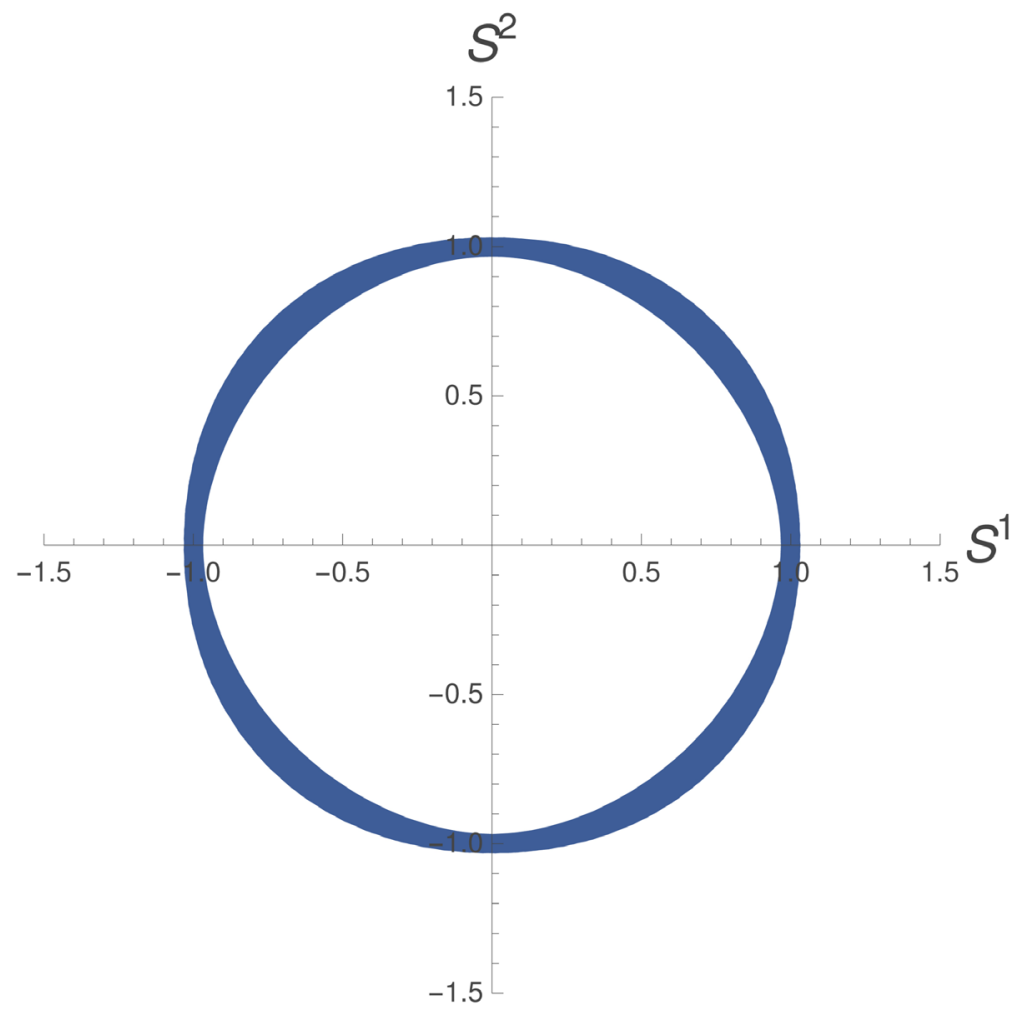

Figure 10. $\frac{C_{11}}{2}=0.025, k z=0, \frac{\omega_{g}}{\omega}=1.02, \quad t=50 T$. 


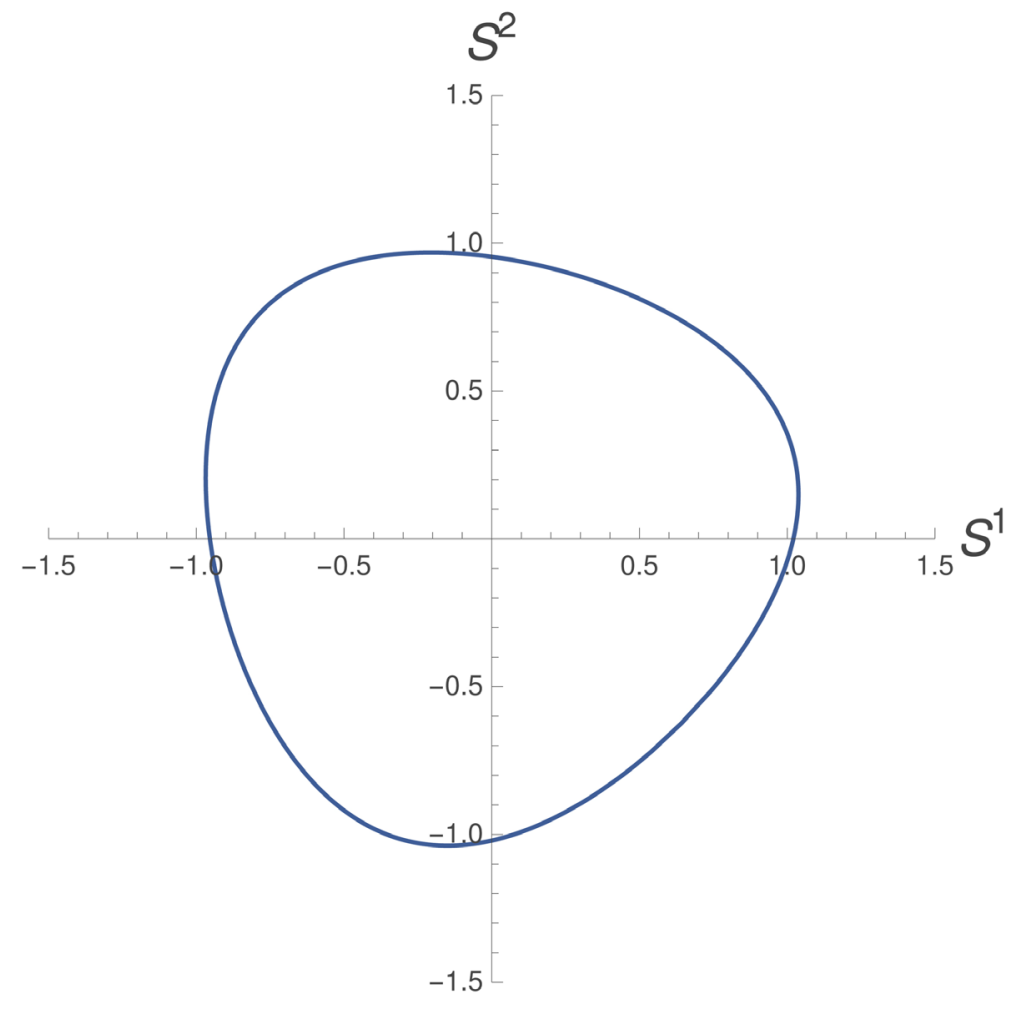

Figure 11. $\frac{C_{11}}{2}=0.05, k z=\pi / 4, \frac{\omega_{g}}{\omega}=1, t=T$.

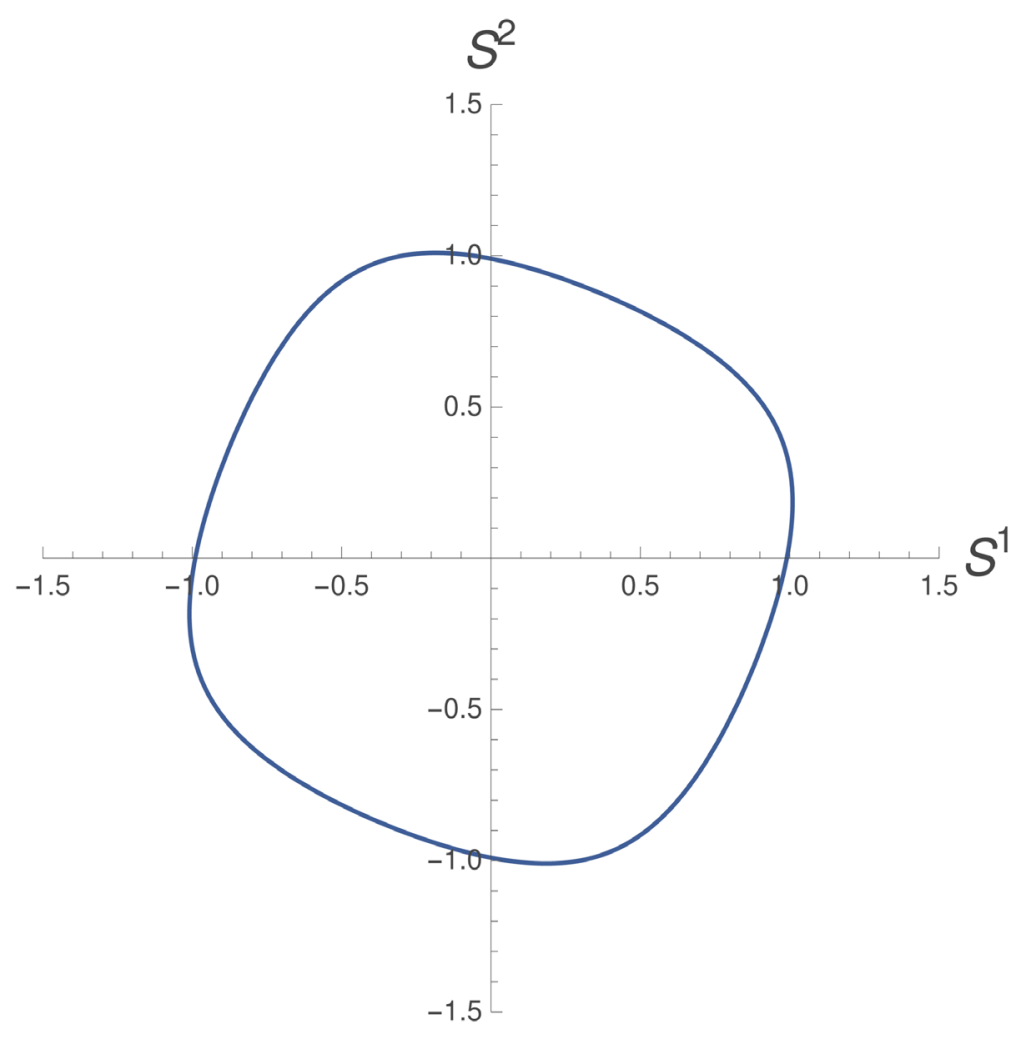

Figure 12. $\frac{C_{11}}{2}=0.05, k z=\pi / 4$ and $\frac{\omega_{g}}{\omega}=2, t=T$. 
$T=\frac{2 \pi}{\omega}$, see Figures 3-8 and Figure 11 and Figure 12. For ratios $\omega_{g} / \omega$ which can be expressed by ratios of small integers the orbits close after several revolutions. With $\frac{\omega_{g}}{\omega}=1.25$ the path does not close yet after 2 revolutions in Figure 7, but it would after 4. In Figures 8-10, we choose $\frac{\omega_{g}}{\omega}=2.1$ and 1.02 respectively and need 10 and 50 revolutions to get a closed paths. In Figure 9 and Figure 10, we compare with different values for the amplitude $C_{11}$. In Figure 11 and Figure 12, we modify the synchronisation of oscillation and rotational motion by choosing an additional phase, $k z=\pi / 4$, leading to a rotation of the diagram compared to Figure 3 and Figure 4 . Due to the periodicity of $h_{11}(t)$ the average radius of the orbits is not modified by gravitational waves. For irrational ratios $\omega_{g} / \omega$ of frequencies, gravitational waves lead to oscillations of the orbits with random phases around the unperturbed circles, to some type of uncertainty of the orbits.

The time-dependence of radial velocities originating in the gravitational waves leads to Coriolis forces and to angular velocities oscillating periodically around the unperturbed value $\omega$. Using Equations (13)-(20) we determine the influence of gravitational waves on angular momentum from the factor

$$
\begin{aligned}
S^{1} U^{2}-S^{2} U^{1} & =\underbrace{R_{1} R_{2}}_{r^{2}}\left(\dot{\varphi}_{2} \cos \varphi_{1} \cos \varphi_{2}+\dot{\varphi}_{1} \sin \varphi_{1} \sin \varphi_{2}\right)-\partial_{0} \frac{h_{11}}{2} \underbrace{2 \cos \varphi_{1} \sin \varphi_{2}}_{\sin (2 \omega t)} \\
& \approx r^{2} \omega+h_{11} r^{2} \omega \cos (2 \omega t)-\partial_{0} \frac{h_{11}}{2} \sin (2 \omega t) .
\end{aligned}
$$

The first term relates to the angular momentum of circular motion. The second term is due to a direct modification of the angular velocity by gravitational waves which for + polarisation contributes proportional to $\cos (2 \omega t)$. The third term of Equation (43) is the result of Coriolis forces modifying the angular momentum due to the contribution of gravitational waves to radial velocities. The first and third term respect the second law of Kepler. The second term gives rise to a deviation from this law, to a time-dependent oscillation of angular momentum due to gravitational waves.

The distance vector $S^{\mu}$ of Equations (13)-(16) was determined for linear polarisation of +-type only, i.e. $C_{11} \neq 0$ and $C_{12}=0$. For arbitrary linear polarisations, $C_{11}^{\prime}=\cos (2 \alpha) C_{11}, C_{12}^{\prime}=\sin (2 \alpha) C_{11}$, the figures have to be rotated by $\alpha$, where the factor 2 reflects the spin 2 property of gravitational waves.

\section{Conclusions}

In this work, we attempted to investigate the influence of gravitational waves on particles circulating in a central force field. We especially concentrated on periodic orbits and their perturbations.

After the introduction, we recapitulated in Section 2 the very well known 
approximate solution for slowly moving particles subjected to gravitational waves with frequency $\omega_{g}$. In Section 3, we expanded the problem to particles circulating with frequency $\omega$ in a central force field. To get a precision of the same order as for slowly moving test particles in Refs. [29]-[34], we had to take into account the centripetal force and to modify the time dependence of the azimuthal angle $\varphi=\omega t$ to $\phi$ of Equation (42). Section 4 is devoted to the graphical representation of the results. Besides the amplitude of the gravitational wave, the ratio $\omega_{g} / \omega$ plays an important role. For integer values, we get periodic orbits. It is amusing to see that these figures have shapes similar to some orbits of electrons in the Bohr model as they are depicted in some class books. For non-integer values of $\omega_{g} / \omega$, the paths are disturbed. For the shape of these perturbations, we have to take into consideration that incident gravitational waves will have different polarisations and therefore different positions of the nodes. In any case, a background of gravitational waves creates a kind of uncertainty in rotating systems.

In further work, it may be interesting to investigate particles circulating on more general elliptical orbits and gravitational waves incidenting from different directions. In addition, a continous spectrum of gravitational waves could be considered.

The idea to this work is inspired by the silicon oil drop experiment of Yves Couder and his group. This is the only experiment, we know, which could give some idea why we can describe nature perfectly by quantum mechanics. A silicon droplet bouncing on a vibrating fluid bath creates waves interfering with the background field [37] created by the vibrations of the fluid. The resonant interaction of particle and field creates a wave field guiding moving droplets. This paper is discussing the question whether gravitational waves could form a similar background influencing electrons on a circular path.

Thinking about the nature of a subquantum medium which could guide elementary particles, we observe the importance of the Compton wave length which is related to the mass of particles. A natural type of background waves which could feel the mass of particles are gravitational waves. Waves which are not in resonance would lead to disturbances and to uncertainties in the position and momentum as we have seen in the investigation presented above. To get closer to quantum mechanics, it would be necessary that the size of these displacements is related to the Compton wavelength. In the above linear treatment, we find closed orbits for any integer ratio $\omega_{g} / \omega$. This does not agree with Bohr's quantisation condition. It would be interesting to take into account the non-linear terms of Einstein's equation and to investigate whether we get a relation to Bohr's quantisation condition.

\section{Acknowledgements}

We thank Alexei A. Deriglazov and Jorge Daniel Casaleiro Lopes for valuable comments. 


\section{Conflict of Interests}

The authors declare that there are no conflicts of interest regarding the publication of this paper.

\section{References}

[1] Einstein, A. (2005) Annalen der Physik, 49, 769-822. https://doi.org/10.1002/andp.19163540702

[2] Abbott, B.P., Abbott, R., Abbott, T.D., Abernathy, M.R., Acernese, F., Ackley, K., Adams, C., Adams, T., Addesso, P., Adhikari, R.X., et al. (2016) Physical Review Letters, 116, Article ID: 061102. https://doi.org/10.1103/PhysRevLett.116.061102

[3] Allen, B. and Romano, J.D. (1999) Physical Review, D59, Article ID: 102001. https://doi.org/10.1103/PhysRevD.59.102001

[4] Misner, C.W., Thorne, K.S. and Wheeler, J.A. (1973) Gravitation. W. H. Freeman, San Francisco.

[5] Hulse, R.A. and Taylor, J.H. (1975) The Astrophysical Journal, 195, L51-L53. https://doi.org/10.1086/181708

[6] Taylor, J.H., Fowler, L.A. and McCulloch, P.M. (1979) Nature, 277, 437-440. https://doi.org/10.1038/277437a0

[7] Weisberg, J.M., Nice, D.J. and Taylor, J.H. (2010) Astrophysical Journal, 722, 1030-1034. https://doi.org/10.1088/0004-637X/722/2/1030

[8] Weisberg, J.M. and Huang, Y.P. (2016) Astrophysical Journal, 829, 55. https://doi.org/10.3847/0004-637X/829/1/55

[9] Detweiler, S.L. (1979) Astrophysical Journal, 234, 1100-1104. https://doi.org/10.1086/157593

[10] Kramer, M. (1998) Astrophysical Journal, 509, 856. https://doi.org/10.1086/306535

[11] Kramer, M., et al. (2006) Science, 314, 97-102. https://doi.org/10.1126/science.1132305

[12] Burgay, M., et al. (2003) Nature, 426, 531-533. https://doi.org/10.1038/nature02124

[13] Hannam, M., Husa, S., Sperhake, U., Bruegmann, B. and Gonzalez, J.A. (2008) Physical Review D, 77, Article ID: 044020. https://doi.org/10.1103/PhysRevD.77.044020

[14] Santamaria, L., et al. (2010) Physical Review D, 82, Article ID: 064016. https://doi.org/10.1103/PhysRevD.82.064016

[15] Baker, J.G., Centrella, J., Choi, D., Koppitz, M. and van Meter, J. (2006) Physical Review Letters, 96, Article ID: 111102. https://doi.org/10.1103/PhysRevLett.96.111102

[16] Bruegmann, B., Tichy, W. and Jansen, N. (2004) Physical Review Letters, 92, Article ID: 211101. https://doi.org/10.1103/PhysRevLett.92.211101

[17] Campanelli, M., Lousto, C.O., Marronetti, P. and Zlochower, Y. (2006) Physical Review Letters, 96, Article ID: 111101. https://doi.org/10.1103/PhysRevLett.96.111101

[18] Connaughton, V., et al. (2016) The Astrophysical Journal, 826, L6. https://doi.org/10.3847/2041-8205/826/1/L6

[19] Belczynski, K., Holz, D.E., Bulik, T. and O'Shaughnessy, R. (2016) Nature, 534, 512-515. https://doi.org/10.1038/nature18322

[20] Altamirano, D., et al. (2011) The Astrophysical Journal, 742, L17. 
https://doi.org/10.1088/2041-8205/742/2/L17

[21] James, O., von Tunzelmann, E., Franklin, P. and Thorne, K.S. (2015) Classical and Quantum Gravity, 32, Article ID: 065001.

https://doi.org/10.1088/0264-9381/32/6/065001

[22] Khriplovich, I.B. and Pomeransky, A.A. (1998) Journal of Experimental and Theoretical Physics, 86, 839-849. https://doi.org/10.1134/1.558554

[23] Deriglazov, A.A. and Guzmn Ramrez, W. (2015) Physical Review D, 92, Article ID: 124017. https://doi.org/10.1103/PhysRevD.92.124017

[24] Deriglazov, A.A. and Guzmn Ramrez, W. (2016) Advances in High Energy Physics, 2016, Article ID: 1376016.

[25] Deriglazov, A.A. and Guzmn Ramrez, W. (2017) Advances in Mathematical Physics, 2017, Article ID: 7397159.

[26] Zhang, P.M., Duval, C. and Horvathy, P.A. (2017) Memory Effect for Impulsive Gravitational Waves.

[27] Zhang, P.M., Duval, C., Gibbons, G.W. and Horvathy, P.A. (2017) Physical Review $D$, 96, Article ID: 064013. https://doi.org/10.1103/PhysRevD.96.064013

[28] Zhang, P.M., Duval, C., Gibbons, G.W. and Horvathy, P.A. (2017) Physics Letters B, 772, 743-746. https://doi.org/10.1016/j.physletb.2017.07.050

[29] Carroll, S.M. (2004) Spacetime and Geometry: An Introduction to General Relativity. Addison Wesley, Boston.

[30] Cheng, T.-P. (2005) Relativity, Gravitation and Cosmology. A Basic Introduction. Oxford University Press, Oxford.

[31] Schroder, U.E. (2007) Gravitation: Einfuhrung in die allgemeine Relativitatstheorie. Deutsch.

[32] Sharan, P. (2009) Space-Time, Geometry and Gravitation. Progress in Mathematical Physics, Springer Verlag, New York. https://doi.org/10.1007/978-3-7643-9971-9

[33] Braccini, S. and Fidecaro, F. (2016) The Detection of Gravitational Waves. Springer International Publishing, Cham, 237. https://doi.org/10.1007/978-3-319-20224-2_7

[34] Rebhan, E. (2011) Theoretische Physik: Relativitatstheorie und Kosmologie. Theoretische Physik/Eckhard Rebhan. Spektrum Akademischer Verlag.

[35] Will, C.M. (2016) Gravity: Newtonian, Post-Newtonian, and General Relativistic. Springer International Publishing, Cham, 9-72.

[36] Pereira, J.G. (2013) Gravitational Waves: A Foundational Review.

[37] Couder, Y. and Fort, E. (2006) Physical Review Letters, 97, Article ID: 154101. https://doi.org/10.1103/PhysRevLett.97.154101 